\title{
Theoretical analysis of phonon dynamical behaviour of Cesium Chloride at various temperatures
}

\author{
S.K.Shukla ${ }^{1}$, K.K.Mishra ${ }^{2 *}$, A.N. Pandey ${ }^{1}$, G.K.Upadhyay ${ }^{2}$ and K.S.Upadhyaya ${ }^{1}$ \\ ${ }^{I}$ Department of Physics, Nehru Gram Bharati University, Kotwa, Jamunipur, Jhusi, Allahabad-221505 \\ ${ }^{2}$ Solid Materials Research Lab, Landmark Technical Campus, Didauli, NH-24, J.P.Nagar, U.P.244222
}

\begin{abstract}
We have developed a new model to investigate the complete lattice dynamics of cesium chloride at $78 \mathrm{~K}$ and at room temperature (298 K). The new model, van der Waals three body force shell model (VTSM) incorporates the effect of van der Waals interactions and three-body interactions in the frame work of rigid shell model where short range interactions are effective up to the second neighbour. A good agreement has been obtained between theory and experiment for dependant properties also.
\end{abstract}

Keywords: Lattice dynamics, Phonon dispersion curves, Debye temperature, two phonon IR and Raman Spectra, Anharmonic elastic constants, combined density of states.

\section{Introduction}

Cesium halides have been given a keen interest to most of the workers in the field of lattice dynamics due to the paramount importance of such binary solids, as the nature and physical properties of such crystal reveals to an understanding of their vibrational, thermo-dynamical, elastic, optical, thermal and numerous physical properties. The availability of a lot of measured data on elastic constants [1-4], dielectric constants [5,7], phonon dispersion curves [6,7,8], Debye temperature variations [9-11], two phonon IR and Raman spectra [12-15], third and fourth order elastic constants and their pressure derivatives [16,17], for all of them $(\mathrm{CsCl}$, $\mathrm{CsBr}$, CsI) and their interpretations by means of theoretical models [18-25] with moderate success, has motivated the present author to the basic need for a lattice dynamical model for the satisfactory description of their interesting properties.

Various experimental and theoretical workers have given evolution for the study of phonon behaviour of $\mathrm{CsCl}$ structure. The first and most important model considered the ions of the crystal to be rigid, undeformable and unpolarizable spherical particle is rigid ion model (RIM) of Kellermann [25] which could not interpret well the dynamical, optical and elastic properties. Further efforts have been put as deformation dipole model (DDM) of Karo and Hardy [19] and rigid shell model (RSM) of Dick and Overhauser [26] and Woods et al. [23] by two different groups of workers. The DDM allows only the redistribution of charges in deformed electron cloud while the shell model considers the relative displacement. Therefore both effects (deformation and displacement) are present in ionic crystals. A general way to remove this deficiency is to include the deformation of electron shells in the framework of RSM. The most prominent amongst them are breathing shell model (BSM) of Schroder [20], the deformable shell model [DSM] of Basu and Sengupta [21], three-body force shell model (TSM) of Verma and Singh [27] and further Singh et al. [1] used extended three-body-force shell model (ETSM) which is an amalgamation of RSM and DDM.

It is emergent from the descriptions that the most realistic model for the lattice dynamics and statics of these crystals can be developed by introducing the effect of van der Waals interactions (VWI), and three body interactions (TBI) in the framework of RSM, where the short-range interactions have been considered up to the second neighbours. The development of such a lattice dynamical model is the chief aim of the present work. This model is known as van der Waals three body force shell model (VTSM).

A formal description of VWI and three-body interactions in the framework of RSM has been described in section 2. The present model VTSM in a way as outline above has been applied to investigate the complete lattice dynamics of cesium halides $(\mathrm{CsCl}, \mathrm{CsBr}, \mathrm{CsI})$. The motivation behind the choice of the present system of solids lies in the fact that they are characterized by high energy gap, some discrepancies in PDCs and Cauchy violations.

The effect of TBI and VWI are quite significant and play a vital role in the description of lattice dynamics of cesium halides. The proposed investigations have been carried out by adopting a simple method to determine a consistent set of 14-parameters $\left[b, \rho, f\left(r_{0}\right), r_{0} f^{\prime}\left(r_{0}\right), A_{11}, A_{22}, A_{12}, B_{11}, B_{22}, B_{12}, d_{1}, d_{2}, Y_{1}\right.$ and $\left.Y_{2}\right]$ of VTSM. In the present paper, we have calculated the phonon dispersion curve of cesium chloride at $78 \mathrm{~K}$ and room temperature $(298 \mathrm{~K})$.

Moreover, we have tested the adequacy of the present model by calculating the two phonon Raman spectra, Debye temperature at $78 \mathrm{~K}$ and $298 \mathrm{~K}$, combined density of states at78 K and $298 \mathrm{~K}$, TOEC and the pressure derivatives of SOEC and TOEC. The theoretical results thus obtained have been compared with 
experimental data and found to show a reasonably good agreement. On the basis of its overall success our model VTSM can be considered as a versatile model for the crystal dynamics of cesium halides.

\section{Theory}

In addition to the Coulomb attraction force, there exists another attraction between the closed shell atoms. The nature of this attraction is different and owes its origin to the correlations of the electron motions in different atoms. The electrons of each atom shift with respect to the nucleus in the presence of other atoms and consequently an atom becomes an electric dipole. The instantaneous dipole moment of a closed shell atom induces a dipole moment on a similar atom and the interactions energy thus arising, is known as the van der Waals interaction (VWI) potential. Now after inclusion of the effects of van der Waals interactions and threebody interactions in the frame work of both ions Polarizable rigid shell model (RSM), the general formalism of present model (VTSM) can be derived from the crystal potential whose relevant expression per unit cell is given by

$$
\Phi(\mathrm{r})=\Phi_{\mathrm{LR}}(r)+\Phi_{S R}(r)
$$

where the first term $\Phi_{\mathrm{LR}}(r)$ represents the long-range Coulomb and three body interaction (TBI) energies expressed by

$$
\Phi_{\mathrm{LR}}(r)=\sum_{i j} \frac{Z_{i} Z_{j} e^{2}}{r_{i j}}\left\{1+\sum_{i j k} f\left(r_{i k}\right)\right\}=\frac{\alpha_{M} Z^{2} e^{2}}{r}\left\{1+\frac{16}{Z} f(r)\right\}
$$

where $\mathrm{Z}$ is the ionic charge parameter of $\mathrm{i}^{\text {th }}$ ion, $\mathrm{r}_{\mathrm{ij}}$ separation between $\mathrm{i}^{\text {th }}$ and $\mathrm{j}^{\text {th }}$ ion, $\mathrm{f}\left(\mathrm{r}_{\mathrm{ik}}\right)$ is the three body force parameter dependent on nearest-neighbour separation $r_{i k}$ and is a measure of ion size difference [23] and $\alpha_{M}$ is Madelung constant ( -1.762670$)$.

The second term in (1) is short-range energy contributions from overlap repulsion and van der Waals interactions (vdWI) expressed as [23].

$$
\Phi_{\mathrm{SR}}(r)=N b \sum_{i, j=1}^{2} \beta_{i j} \exp \left[r_{i}+r_{j}-r_{i j} / \rho\right]+\sum_{i j} \frac{c_{i j}}{r_{i j}^{6}}+\sum_{i j} \frac{d_{i j}}{r_{i j}^{8}}
$$

where the first term is the Hafemeister and Flygare (HF) potential [28]. The second term and third term represent the energy due to vdW dipole-dipole (d-d) and dipole-quadrupole (d-q) interactions, respectively. written as:

Using the crystal energy expression (1), the equations of motion of two cores and two shells can be

$$
\begin{aligned}
& \omega^{2} \underline{M} \underline{U}=\left(\underline{R}+\underline{Z}_{m} \underline{C^{\prime}} \underline{Z}_{\underline{m}}\right) \underline{U}+\left(\underline{T}+\underline{Z}_{\underline{m}} \frac{C^{\prime}}{C^{\prime}} \underline{\underline{Y}}\right) \underline{W} \\
& O=\left(\underline{T}^{T}+\underline{Y}_{m} \underline{C}^{\prime} \underline{Z}_{\underline{m}}\right) \underline{\underline{U}}+\left(\underline{S}+\underline{K}+\underline{Y}_{\underline{m}} \underline{C^{\prime}} \underline{Y}_{\underline{m}}\right) \underline{W}
\end{aligned}
$$

Here $\underline{U}$ and $\underline{W}$ are vectors describing the ionic displacements and deformations, respectively. $\underline{Z}_{\underline{m}}$ and $\underline{Y}_{\underline{m}}$ are diagonal matrices of modified ionic charges and shell charges, respectively. The elements of matrix $\underline{Z}_{m}$ consists of the parameter $\underline{Z}_{m}$ thereby giving the modified ionic charge.

$$
Z_{m}=\xi= \pm Z \sqrt{1+(12 / Z) f_{0}}
$$

The elimination of $\underline{W}$ from eqns. (6) and (7) leads to the secular determinant:

$$
\left|\underline{D}(\vec{q})-\omega^{2} \underline{M} \underline{I}\right|=0
$$

for the frequency determination. Here $\underline{D}(q)$ is the $(6 \times 6)$ dynamical matrix given by

$$
\begin{aligned}
\underline{D}(\vec{q})= & \left(\underline{R}^{\prime}+\underline{Z}_{m} \underline{C}^{\prime} \underline{Z}_{m}\right)-\left(\underline{T}+\underline{Z}_{m} \underline{C}^{\prime} \underline{Y}_{m}\right) \times \\
& \left(\underline{S}+\underline{K}+\underline{Y}_{m} \underline{C}^{\prime} \underline{Y}_{m}\right)^{-1}\left(\underline{T}^{T}+\underline{Y}_{m} \underline{C}^{\prime} \underline{Z}_{m}\right)
\end{aligned}
$$

The numbers of adjustable parameters have been largely reduced by considering all the short-range interactions to act only through the shells.

The expressions derived for elastic constants from eqn. (7) corresponding to VTSM are obtained as

$$
\begin{aligned}
& C_{11}=\frac{e^{2}}{4 a^{4}}\left[0.7010 Z_{m}{ }^{2}+\frac{A_{12}+2 B_{12}}{6}+\frac{A_{11}+A_{22}}{4}+5.4283 \xi^{\prime 2}\right] \\
& C_{12}=\frac{e^{2}}{4 a^{4}}\left[-0.6898 Z_{m}{ }^{2}+\frac{A_{12}-4 B_{12}}{6}-\frac{B_{11}+B_{22}}{4}+5.4283 \xi^{\prime 2}\right] \\
& C_{44}=\frac{e^{2}}{4 a^{4}}\left[-0.3505 Z_{m}{ }^{2}+\frac{A_{12}+2 B_{12}}{6}+\frac{B_{11}+B_{22}}{4}\right]
\end{aligned}
$$

In view of the equilibrium condition $\left[(d \Phi / d r)_{0}=0\right]$ we obtain

$$
B_{11}+2 B_{12}+B_{22}=-0.6786 \mathrm{Zm}^{2}
$$


where $\quad Z_{m}^{2}=Z^{2}\left(1+\frac{16}{Z} f_{0}\right)$ and $\xi^{\prime 2}=Z r_{0} f_{0}^{\prime}$

$f_{0}^{\prime}=(d f / d r)_{r=r_{0}}, \quad r_{0}=a \sqrt{3}$ is the inter-ionic separation.

The term $f_{0}$ is a function dependent on the overlap integrals of the electron wave functions and the subscript zero indicates the equilibrium value. By solving the secular eqn. (7) along [q 00 ] direction and subjecting the short and long-range coupling coefficients to the long-wavelength limit $\vec{q} \rightarrow 0$, two distinct optical vibration frequencies are obtained as

$$
\begin{aligned}
& \left(\mu \omega_{L}^{2}\right)_{q=0}=R_{0}^{\prime}+\frac{\left(Z^{\prime} e\right)^{2}}{\mathrm{v} f_{L}} \cdot \frac{8 \pi}{3}\left(1+12 Z_{m}^{-2} Z r_{0} f_{0}^{\prime}\right) \\
& \left(\mu \omega_{T}^{2}\right)_{q=0}=R_{0}^{\prime}-\frac{\left(Z^{\prime} e\right)^{2}}{\mathrm{v} f_{T}} \cdot \frac{4 \pi}{3}
\end{aligned}
$$

where the abbreviations stand for

$$
\begin{aligned}
& R_{0}^{\prime}=R_{0}-e^{2}\left(\frac{d_{1}^{2}}{\alpha_{1}}+\frac{d_{2}^{2}}{\alpha_{2}}\right) ; Z^{\prime}=Z_{m}+d_{1}-d_{2} \\
& f_{L}=1+\frac{8 \pi \alpha}{3 \mathrm{v}}\left(1+12 Z_{m}^{-2} Z r_{0} f_{0}^{\prime}\right) \\
& f_{T}=1-\frac{4 \pi \alpha}{3 \mathrm{v}} \\
& \text { and } \alpha=\alpha_{1}+\alpha_{2}
\end{aligned}
$$

\section{Result, Discussion And Conclusion}

The calculations on the Cesium halides have been performed with our model VTSM which have fourteen model parameters calculated using above expressions (5-13) and presented along with input data in Table 1. These model parameters have been used to compute the phonon dispersion curves of $\mathrm{CsCl}$ at $78 \mathrm{~K}$ and $298 \mathrm{~K}$. The calculated phonon dispersion curves of $\mathrm{CsCl}$ at $78 \mathrm{~K}$ have been displayed with available experimental and other theoretical data $[6,22]$ in figure 1 and at $298 \mathrm{~K}$ have been traced and compared with available experimental and theoretical data [6] in figure 2. The phonon dispersion curves (PDCs) for all the cesium chloride at both the temperatures are almost similar but there are certain features which deserve special mention. Three-body interactions have influenced both LO and TO branches much more than the acoustic branches (LA and TA) in these halides. Another striking feature of the present model is noteworthy from the excellent reproduction of almost all the acoustic branches. The agreement achieved from the present model is also excellent for the longitudinal acoustic (LA) branch along [qqq] direction. Also the result obtained from present model is almost identical along [qqq] direction. This may be particularly because the zone centre vibration frequencies have been used as input data in the calculation of model parameters. A quantitative interpretation of the general features of PDC is also obvious from the present model when it predicts the gap between the acoustical and optical branches similar to forbidden gap between the valence and the conduction band.

The agreement between our computed phonon spectra and experimental data is excellent in Table 3 for $\mathrm{CsCl}$ at $78 \mathrm{~K}$ and table 4 for $\mathrm{CsCl}$ at $298 \mathrm{~K}$. Our model VTSM has successfully explained the phonon anomalies even along ( $q 00)$ and (qqq) directions. From figure 1, figure 2, table 3 and table 4 it is clear that VTSM, improves result of $\mathrm{SM}$ used calculation of $\mathrm{CsCl}$ at $78 \mathrm{~K}$ by $3.97 \%$ along $\mathrm{T} O(\mathrm{X}), 3.07 \%$ along $L A(\mathrm{X}), 2.16 \%$ along TA (X), $0.26 \%$ along $\mathrm{LO}(\mathrm{R}), 0.52 \%$ along $\mathrm{TO}(\mathrm{R})$ and $2.4 \%$ along $L A(\mathrm{R})$ and $\mathrm{TA}(\mathrm{R})$ and improve the results of TSM used for the calculation of $\mathrm{CsCl}$ at $298 \mathrm{~K}$ by $0.887 \%$ along $\mathrm{L} O(\mathrm{X}), 6.9767 \%$ along $L A(\mathrm{X})$, 9.022\% along TA (X), $1.0781 \%$ along $\mathrm{LO}(\mathrm{R}), 1.33 \%$ along $\mathrm{TO}(\mathrm{R})$ and $3.94 \%$ along $\mathrm{TA}(\mathrm{R})$. The deviation between theory and experimental due to $S M$ is maximum $4.61 \%$ along $L O(\mathrm{X})$ while due VTSM the deviation is maximum $0 \%$ along $L O(\mathrm{X})$. In view of overall success, it may be concluded that our model provides a good agreement between theory and experiment and is certainly better than those yielded by theoretical workers [1825]. It is obvious from these tables that the frequencies at $\mathrm{X}, \mathrm{R}$ and $\mathrm{M}$ points reported from present model VTSM are very much close to the experimental values [6].

As the two-phonon Raman and infra-red (IR) spectra are sensitive to the higher frequency side of the phonon spectra and the specific heats are sensitive to its lower side. It seems, therefore, essential to investigate the extent to which the present model is capable to interpret the Raman and IR spectra. This will provide a complete test for the validity of the present model for entire range of the spectra. The result of these investigations from CDS approach depicted in figure 2 shows that the agreement between experimental $[14,15]$ 
and our theoretical peaks is generally good for both Raman and IR spectra in almost all the cesium halides. Since our CDS curves are derived from somewhat course mesh division of the Brillouin zone, therefore, we have used a critical points analysis [29] to present complex interpretation of the observed Raman spectra. The assignments made by the present study listed in table 3 shows reasonably good agreement with the observed peaks corresponding to Raman and IR spectra for $\mathrm{CsCl}$ at both the temperatures. Our extensive study of twophonon Raman and IR spectra is basically aimed to correlate the theoretical and optical experimental results. The interpretation of Raman and IR spectra achieved from both CDS approach and critical points analysis may be considered satisfactory in all cases. The variation of Debye temperatures $\left(\Theta_{\mathrm{D}}\right)$ with the temperature $(\mathrm{T})$ are shown in the figure 4 for $\mathrm{CsCl}(78 \mathrm{~K})$ and $(298 \mathrm{~K})$. The calculated $\left(\Theta_{\mathrm{D}}-\mathrm{T}\right)$ curves for $\mathrm{CsCl}$ at $78 \mathrm{~K}$ and $298 \mathrm{~K}$ are in excellent agreement with the experimental results [9-11]. Though the agreement is almost better with our model VTSM, still, there is slight discrepancy between theoretical and experimental results at higher temperatures which can be due to the exclusion of the effect of anharmonicity in the present model. This success of present model in exploring the specific heat indicates its adequacies to describe the lower range of frequency spectra. This is not surprising because the present model is quite capable to explain the elastic constants and hence the acoustic frequencies in terms of three-body interactions.

It is interesting to note that our results on TOEC, pressure derivatives of SOEC and Cauchy discrepancy are generally better than those of others as is evident from the tables 6,7 , and 8 . However, the results are closer to their experimental values $[16,17]$. It can be seen from table 8 that the Cauchy discrepancy is smaller for TOE constant than for SOE constants. A possible explanation for this fact seems to be that manybody and/or thermal effects are more pronounced for SOEC than for TOEC. The pressure derivatives of the effective SOE constants calculated by us have been given in table 7 and found to be generally in good agreement with their observed data.

In view of the overall achievements described above, it may be concluded that the modification introduced by TBI and VWI in the frame work of RSM is very much importance in the crystals under considerations. In fact, the present model VTSM has revealed much better descriptions of dynamical properties of these compounds than those obtained by rigid shell model [26], deformation shell model [19], breathing shell model [20], extended three-body force shell model [1]. This study gets strong support from recent publications of our research group [32-43]. The induction of van der Waals interactions is much essential in the study of lattice dynamics of alkali halides crystals.

\section{IV. $\quad$ Figures And Tables}

Table 1: Input data and model parameters for $\operatorname{CsCl}(78 \mathrm{~K})\left[C_{i j}\right.$ and $\mathrm{B}\left(\right.$ in $\left.10^{11} \mathrm{dyne} / \mathrm{cm}^{2}\right), \quad v\left(\right.$ in $\left.10^{13} \mathrm{~Hz}\right)$, $r_{0}\left(\right.$ in $\left.10^{-8} \mathrm{~cm}\right), \alpha_{i}\left(\right.$ in $\left.10^{-24} \mathrm{~cm}^{3}\right), \mathrm{b}\left(\right.$ in $10^{-12} \mathrm{erg}$ ), $\rho$ (in $\left.\left.10^{-8} \mathrm{~cm}\right),\right]$

\begin{tabular}{|c|c|c|c|c|}
\hline \multicolumn{3}{|c|}{ Input Data } & \multicolumn{2}{c|}{ Model Parameters } \\
\hline Properties & Values & References & Parameters & Values \\
\hline $\mathrm{C}_{11}$ & 4.260 & {$[5]$} & $b$ & 0.273 \\
\hline $\mathrm{C}_{12}$ & 1.300 & {$[5]$} & $\rho$ & 0.378 \\
\hline $\mathrm{C}_{44}$ & 1.092 & {$[5]$} & $f\left(r_{0}\right)$ & -3.98 \\
\hline $\mathrm{B}$ & 17.0 & {$[31]$} & $r_{0} f^{\prime}\left(r_{0}\right)$ & 21.5214 \\
\hline$r_{0}$ & 2.044 & {$[1]$} & $\mathrm{A}_{12}$ & 6.5878 \\
\hline$v_{\mathrm{LO}}(\Gamma)$ & $4.90^{*}$ & {$[6]$} & $\mathrm{B}_{12}$ & -0.3863 \\
\hline$v_{\mathrm{TO}}(\Gamma)$ & 3.19 & {$[6]$} & $\mathrm{A}_{11}$ & -0.7240 \\
\hline$v_{\mathrm{LO}}(\mathrm{M})$ & 3.75 & {$[6]$} & $\mathrm{B}_{11}$ & -0.0197 \\
\hline$v_{\mathrm{TO}}(\mathrm{M})$ & 2.79 & {$[6]$} & $\mathrm{A}_{22}$ & 1.4497 \\
\hline$v_{\mathrm{LA}}(\mathrm{M})$ & 1.92 & {$[6]$} & $\mathrm{B}_{22}$ & -0.9242 \\
\hline$v_{\mathrm{TA}}(\mathrm{M})$ & 1.22 & {$[6]$} & $\mathrm{d}_{1}$ & 0.2825 \\
\hline$\alpha_{1}$ & 2.798 & {$[1]$} & $\mathrm{d}_{2}$ & 0.3902 \\
\hline$\alpha_{2}$ & 2.653 & {$[1]$} & $\mathrm{Y}_{1}$ & -2.2482 \\
\hline$\varepsilon_{0}$ & 6.680 & {$[1]$} & $\mathrm{Y}_{2}$ & -1.5432 \\
\hline
\end{tabular}

*Extrapolated 
Theoretical analysis of phonon dynamical behaviour of Cesium Chloride at various temperatures

Table 2: Input data and model parameters for $\mathrm{CsCl}(298 \mathrm{~K})\left[C_{i j}\right.$ and $\mathrm{B}$ (in $\left.10^{11} \mathrm{dyne} / \mathrm{cm}^{2}\right), v\left(\right.$ in $\left.10^{13} \mathrm{~Hz}\right)$, $r_{0}\left(\right.$ in $\left.10^{-8} \mathrm{~cm}\right), \alpha_{i}\left(\right.$ in $\left.10^{-24} \mathrm{~cm}^{3}\right), \mathrm{b}\left(\right.$ in $10^{-12} \mathrm{erg}$ ), $\rho$ (in $\left.\left.10^{-8} \mathrm{~cm}\right),\right]$

\begin{tabular}{|c|c|c|c|c|}
\hline \multicolumn{3}{|c|}{ Input Data } & \multicolumn{2}{c|}{ Model Parameters } \\
\hline Properties & Values & References & Parameters & Values \\
\hline $\mathrm{C}_{11}$ & 4.068 & {$[5]$} & $b$ & 0.249 \\
\hline $\mathrm{C}_{12}$ & 1.200 & {$[5]$} & $\rho$ & 0.311 \\
\hline $\mathrm{C}_{44}$ & 1.100 & {$[5]$} & $f\left(r_{0}\right)$ & -3.28 \\
\hline $\mathrm{B}$ & 17.0 & {$[31]$} & $r_{0} f^{\prime}\left(r_{0}\right)$ & 21.7418 \\
\hline$r_{\mathrm{O}}$ & 2.0615 & {$[1]$} & $\mathrm{A}_{12}$ & 6.5844 \\
\hline$v_{\mathrm{LO}}(\Gamma)$ & $4.93 *$ & {$[6]$} & $\mathrm{B}_{12}$ & -0.3829 \\
\hline$v_{\mathrm{TO}}(\Gamma)$ & 3.03 & {$[6]$} & $\mathrm{A}_{11}$ & -0.8275 \\
\hline$v_{\mathrm{LO}}(\mathrm{M})$ & 3.73 & {$[6]$} & $\mathrm{B}_{11}$ & 0.0338 \\
\hline$v_{\mathrm{TO}}(\mathrm{M})$ & 2.60 & {$[6]$} & $\mathrm{A}_{22}$ & 1.6651 \\
\hline$v_{\mathrm{LA}}(\mathrm{M})$ & 1.94 & {$[6]$} & $\mathrm{B}_{22}$ & -1.0753 \\
\hline$v_{\mathrm{TA}}(\mathrm{M})$ & 1.09 & {$[6]$} & $\mathrm{d}_{1}$ & 0.2975 \\
\hline$\alpha_{1}$ & 2.798 & {$[1]$} & $\mathrm{d}_{2}$ & 0.3820 \\
\hline$\alpha_{2}$ & 2.653 & {$[1]$} & $\mathrm{Y}_{1}$ & -2.0818 \\
\hline$\varepsilon_{0}$ & 6.950 & {$[1]$} & $\mathrm{Y}_{2}$ & -0.2642 \\
\hline
\end{tabular}

*Extrapolated

Table 3. Comparison of frequencies from various sources ( $\mathrm{X}$ and $\mathrm{R}$ points) for $\mathrm{CsCl}$ at $78 \mathrm{~K}$

\begin{tabular}{|c|c|c|c|c|c|c|c|c|c|}
\hline \multirow{2}{*}{$\mathrm{CsCl}$} & \multirow{2}{*}{ Frequencies } & \multirow{2}{*}{$\begin{array}{c}\text { Exp. } \\
{[6]}\end{array}$} & \multicolumn{3}{|c|}{ SM [6] } & \multicolumn{3}{|c|}{ VTSM (Present Study) } & \multirow{2}{*}{$\begin{array}{c}\% \\
\text { improvement } \\
\text { (a-b) }\end{array}$} \\
\hline & & & Values & \pm dev. & $\%(\mathbf{a})$ & Values & \pm dev. & $\%(b)$ & \\
\hline \multirow{4}{*}{$\underset{(100)}{X}$} & LO $(\mathrm{THz})$ & 4.61 & 4.61 & 0 & 0 & 4.61 & 0 & 0 & 0 \\
\hline & TO $(\mathrm{THz})$ & 3.02 & 3.15 & 0.04 & 4.30 & 3.03 & 0.0033 & 0.33 & 3.97 \\
\hline & LA $(\mathrm{THz})$ & 2.60 & 2.68 & 0.03 & 3.07 & 2.60 & 0 & 0 & 3.07 \\
\hline & TA $(\mathrm{THz})$ & 1.39 & 1.35 & 0.0287 & 2.87 & 1.38 & 0.00719 & 0.719 & 2.16 \\
\hline \multirow{4}{*}{$\begin{array}{c}\mathrm{R} \\
(.5 .5 .5)\end{array}$} & LO $(\mathrm{THz})$ & 3.82 & 3.83 & 0.0026 & 0.26 & 3.82 & 0 & 0 & 0.26 \\
\hline & TO $(\mathrm{THz})$ & 3.84 & 3.81 & 0.0078 & 0.78 & 3.83 & 0.0026 & 0.26 & 0.52 \\
\hline & LA $(\mathrm{THz})$ & 2.05 & 2.00 & 0.024 & 2.4 & 2.05 & 0 & 0 & 2.4 \\
\hline & TA $(\mathrm{THz})$ & 2.05 & 2.00 & 0.024 & 2.4 & 2.05 & 0 & 0 & 2.4 \\
\hline
\end{tabular}

Table 4. Comparison of frequencies from various sources ( $\mathrm{X}$ and $\mathrm{R}$ points) for $\mathrm{CsCl}$ at $298 \mathrm{~K}$

\begin{tabular}{|c|c|c|c|c|c|c|c|c|c|}
\hline \multirow{2}{*}{$\mathrm{CsCl}$} & \multirow{2}{*}{ Frequencies } & \multirow{2}{*}{$\begin{array}{c}\text { Exp. } \\
{[6]}\end{array}$} & \multicolumn{3}{|c|}{ ETSM [32] } & \multicolumn{3}{|c|}{ VTSM (Present Study) } & \multirow{2}{*}{$\begin{array}{c}\% \\
\text { improvement } \\
\text { (a-b) }\end{array}$} \\
\hline & & & Values & \pm dev. & $\%(\mathbf{a})$ & Values & \pm dev. & $\%(b)$ & \\
\hline \multirow{4}{*}{$\begin{array}{c}\mathrm{X} \\
(100)\end{array}$} & LO $(\mathrm{THz})$ & 4.51 & 4.55 & 0.00887 & 0.887 & 4.51 & 0 & 0 & 0.887 \\
\hline & TO $(\mathrm{THz})$ & 2.85 & 2.85 & 0 & 0 & 2.85 & 0 & 0 & 0 \\
\hline & LA $(\mathrm{THz})$ & 2.58 & 2.40 & 0.06976 & 6.976 & 2.58 & 0 & 0 & 6.9767 \\
\hline & TA $(\mathrm{THz})$ & 1.33 & 1.21 & 0.09022 & 9.022 & 1.33 & 0 & 0 & 9.022 \\
\hline \multirow{4}{*}{$\begin{array}{c}\mathrm{R} \\
(.5 .5 .5)\end{array}$} & LO $(\mathrm{THz})$ & 3.71 & 3.75 & 0.01078 & 1.078 & 3.71 & 0 & 0 & 1.0781 \\
\hline & TO $(\mathrm{THz})$ & 3.75 & 3.80 & 0.0133 & 1.333 & 3.75 & 0 & 0 & 1.3333 \\
\hline & LA $(\mathrm{THz})$ & 2.01 & 2.00 & 0.00497 & 0.497 & 2.00 & 0.00497 & 0.497 & 0 \\
\hline & TA $(\mathrm{THz})$ & 2.03 & 1.95 & 0.0394 & 3.940 & 2.03 & 0 & 0 & 3.940 \\
\hline
\end{tabular}


Theoretical analysis of phonon dynamical behaviour of Cesium Chloride at various temperatures

Table 5: Assignment of two phonon Raman and Infra-red peaks for CsCl

\begin{tabular}{|c|c|c|c|c|c|c|c|c|c|c|c|}
\hline \multicolumn{6}{|c|}{ Raman and Infra-red peaks for $\mathrm{CsCl}(78 \mathrm{~K})$} & \multicolumn{6}{|c|}{ Raman and Infra-red peaks for $\mathrm{CsCl}(298 \mathrm{~K})$} \\
\hline \multirow[b]{3}{*}{ 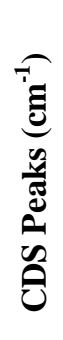 } & \multicolumn{3}{|c|}{ Raman Active } & \multirow{2}{*}{\multicolumn{2}{|c|}{$\begin{array}{c}\text { Infra-red Active } \\
\text { Present Study } \\
\end{array}$}} & \multirow[b]{3}{*}{ 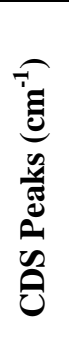 } & \multicolumn{3}{|c|}{ Raman Active } & \multirow{2}{*}{\multicolumn{2}{|c|}{$\begin{array}{c}\text { Infra-red Active } \\
\text { Present Study } \\
\end{array}$}} \\
\hline & \multirow[b]{2}{*}{ 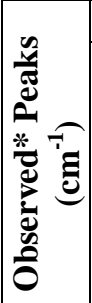 } & \multicolumn{2}{|c|}{ Present Study } & & & & \multirow[b]{2}{*}{ 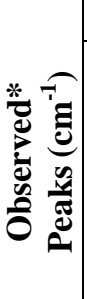 } & \multicolumn{2}{|c|}{ Present Study } & & \\
\hline & & 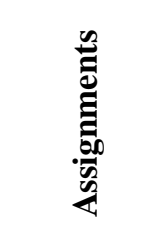 & 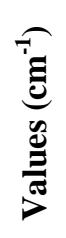 & 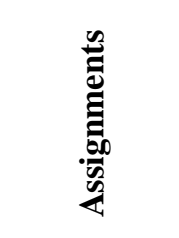 & 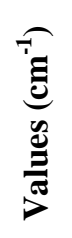 & & & 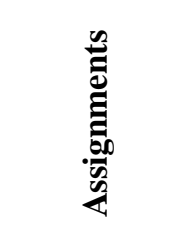 & 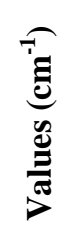 & 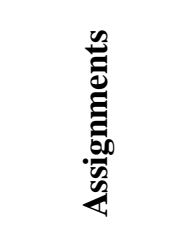 & 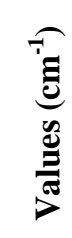 \\
\hline 42 & 42 & LA-TA(X) & 42 & & & 41 & 42 & LA-TA(X) & 41 & & \\
\hline 62 & 61 & LO-LA(X) & 66 & & & 60 & 61 & LO-LA(X) & 61 & & \\
\hline 81 & 81 & $2 \mathrm{TA}(\mathrm{M})$ & 80 & $2 \mathrm{TA}(\mathrm{M})$ & 80 & 80 & 81 & $2 \mathrm{TA}(\mathrm{X})$ & 88 & & \\
\hline 113 & 112 & LO-TA(X) & 108 & & & 113 & 112 & LO-TA(X) & 107 & & \\
\hline 158 & 158 & TO-LA(M) & 156 & TO-LA(M) & 156 & 153 & 158 & TO+LA(M) & 151 & $\mathrm{TO}+\mathrm{LA}(\mathrm{M})$ & 151 \\
\hline 170 & 170 & $2 \mathrm{LA}(\mathrm{X})$ & 174 & & & 173 & 170 & $2 \mathrm{LA}(\mathrm{X})$ & 170 & & \\
\hline 212 & 212 & $2 \mathrm{TO}(\Gamma)$ & 212 & $\mathrm{LO}+\mathrm{TO}(\mathrm{M})$ & 217 & 212 & 212 & $2 \mathrm{TO}(\Gamma)$ & 212 & $\mathrm{LO}+\mathrm{TO}(\mathrm{M})$ & 212 \\
\hline 233 & & $\begin{array}{l}\mathrm{LO}+\mathrm{LA}(\mathrm{X} \\
\mathrm{l}\end{array}$ & 240 & & & & & LO-LA(X) & 236 & & \\
\hline 260 & & $\begin{array}{l}2 \mathrm{LO}(\mathrm{M}) \\
2 \mathrm{LO}(\mathrm{R})\end{array}$ & $\begin{array}{l}251 \\
254\end{array}$ & $2 \mathrm{LO}(\mathrm{M})$ & 251 & & & 2LO(M) & 251 & $2 \mathrm{LO}(\mathrm{M})$ & 251 \\
\hline 310 & 310 & & & & & 310 & 310 & $2 \mathrm{LO}(\mathrm{X})$ & 302 & & \\
\hline 353 & 353 & $2 \mathrm{LO}(\Gamma)$ & 334 & & & 353 & 353 & $2 \mathrm{LO}(\Gamma)$ & 328 & & \\
\hline
\end{tabular}

$*[14,15]$

Table 6. Third order elastic constants (in units $10^{12} \mathrm{dyne} / \mathrm{cm}^{2}$ ) for CsCl.

\begin{tabular}{|c|c|c|}
\hline \multirow{2}{*}{ Property } & TOEC of CsCl at 78 K & TOEC of CsCl at 298 K \\
\cline { 2 - 3 } & Values & Values \\
\hline$C_{111}$ & -0.13820 & -0.18501 \\
\hline$C_{112}$ & -0.2485 & -0.2448 \\
\hline$C_{166}$ & -0.2516 & -0.2477 \\
\hline$C_{123}$ & -0.2369 & -0.2316 \\
\hline$C_{144}$ & -0.2324 & -0.2280 \\
\hline$C_{456}$ & -0.2300 & -0.2280 \\
\hline
\end{tabular}

Table 7. Values of pressure derivatives of SOE constants for CsCl

\begin{tabular}{|c|c|c|c|}
\hline \multirow{2}{*}{ Property } & \multicolumn{3}{|c|}{ Pressure derivative of SOEC for CsCl } \\
\cline { 2 - 4 } & at 78 K & Values & at 298 K \\
\hline & Values & 8.1282 & 3.56 \\
\hline$d k^{\prime} / d P$ & 7.9706 & -0.8027 & 0.89 \\
\hline$d s^{\prime} / d P$ & -0.8932 & 3.5867 & $\ldots \ldots$ \\
\hline$d c_{44}^{\prime} / d P$ & 3.7546 & $30]$ \\
\hline
\end{tabular}

Table 8. The Values of Cauchy discrepancy (in units $10^{12} \mathrm{dyne} / \mathrm{cm}^{2}$ ) for $\mathrm{CsCl}$.

\begin{tabular}{|c|c|c|}
\hline \multirow{2}{*}{ Property } & TOEC of CsCl at 78 K & TOEC of CsCl at 298 K \\
\cline { 2 - 3 } & Values & Values \\
\hline$C_{112}-C_{166}$ & -0.000477 & -0.000427 \\
\hline$C_{123}-C_{456}$ & -0.006919 & -0.003622 \\
\hline$C_{144}-C_{456}$ & -0.002306 & -0.001207 \\
\hline$C_{123}-C_{144}$ & -0.004613 & -0.002415 \\
\hline
\end{tabular}



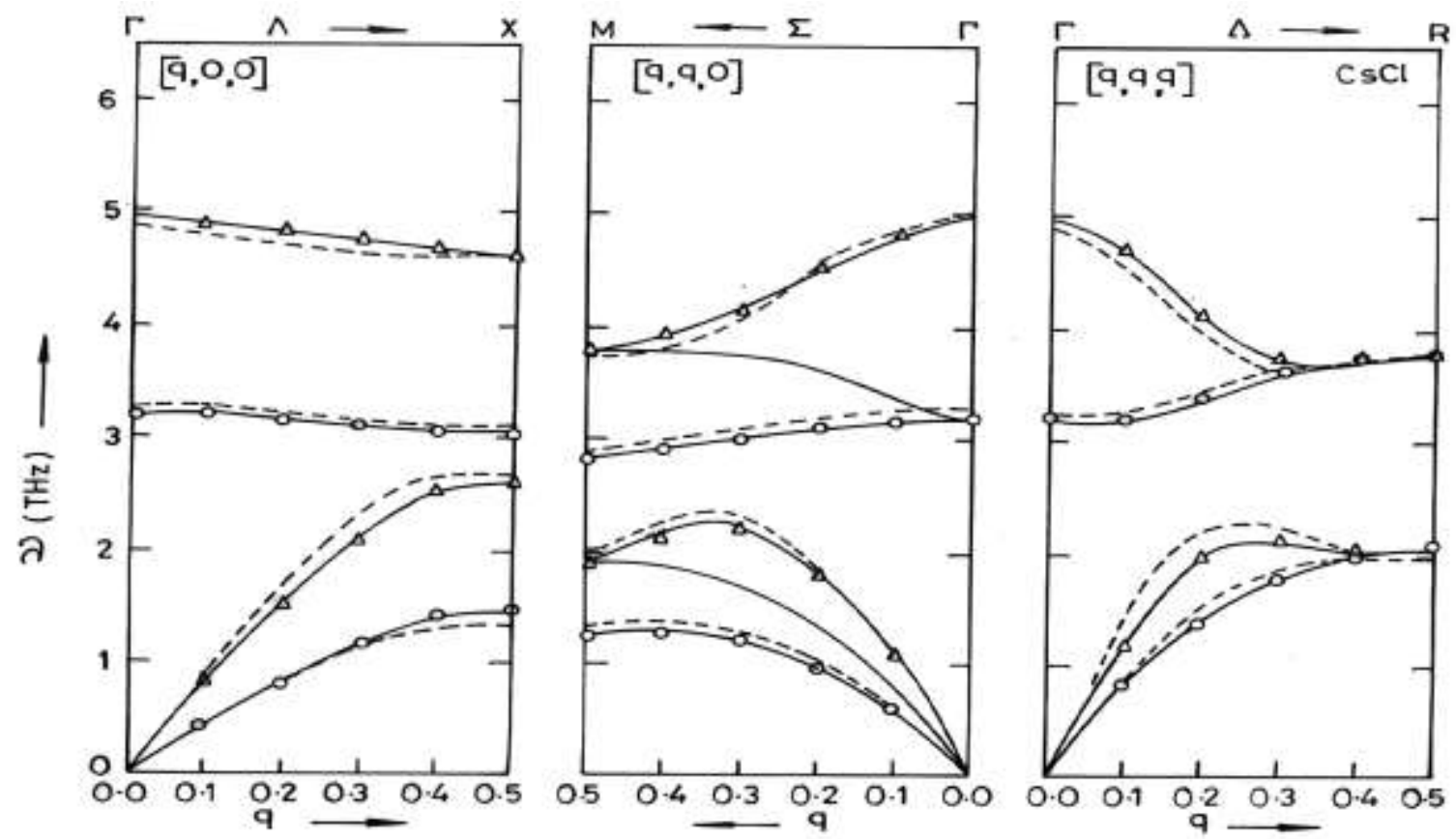

Figure 1: Phonon Dispersion Curve for $\mathrm{CsCl}$ at $78 \mathrm{~K}$

o Transverse

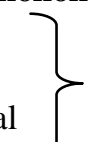
Experimental Points

Present Study

$\Delta$ Longitudinal $\}$ - - - - Shell Model Study
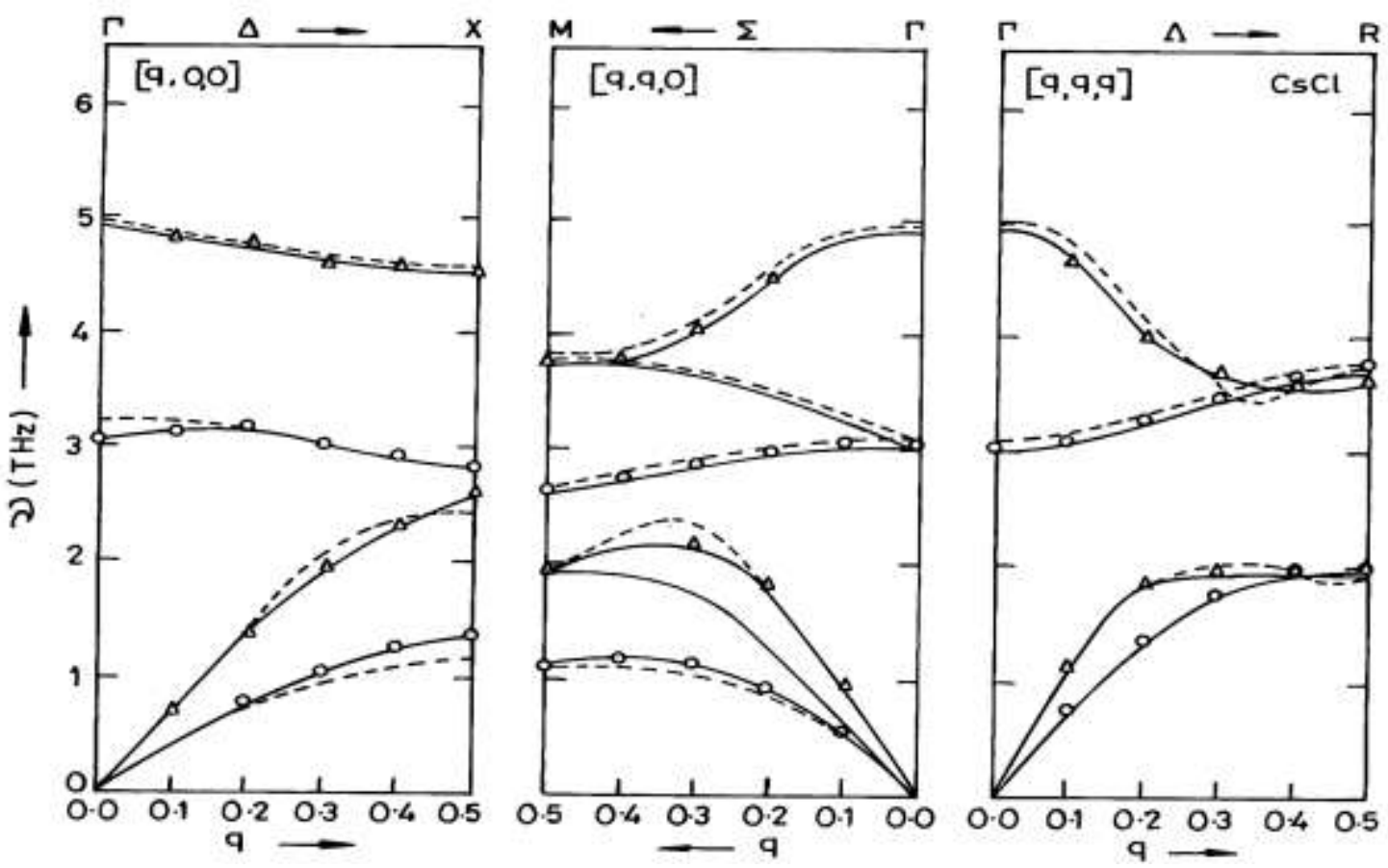

Figure 2: Phonon Dispersion Curve for $\mathrm{CsCl}$ at $298 \mathrm{~K}$
O Transverse
Present Study
$\Delta$ Longitudinal $\}$ Experimental Points 


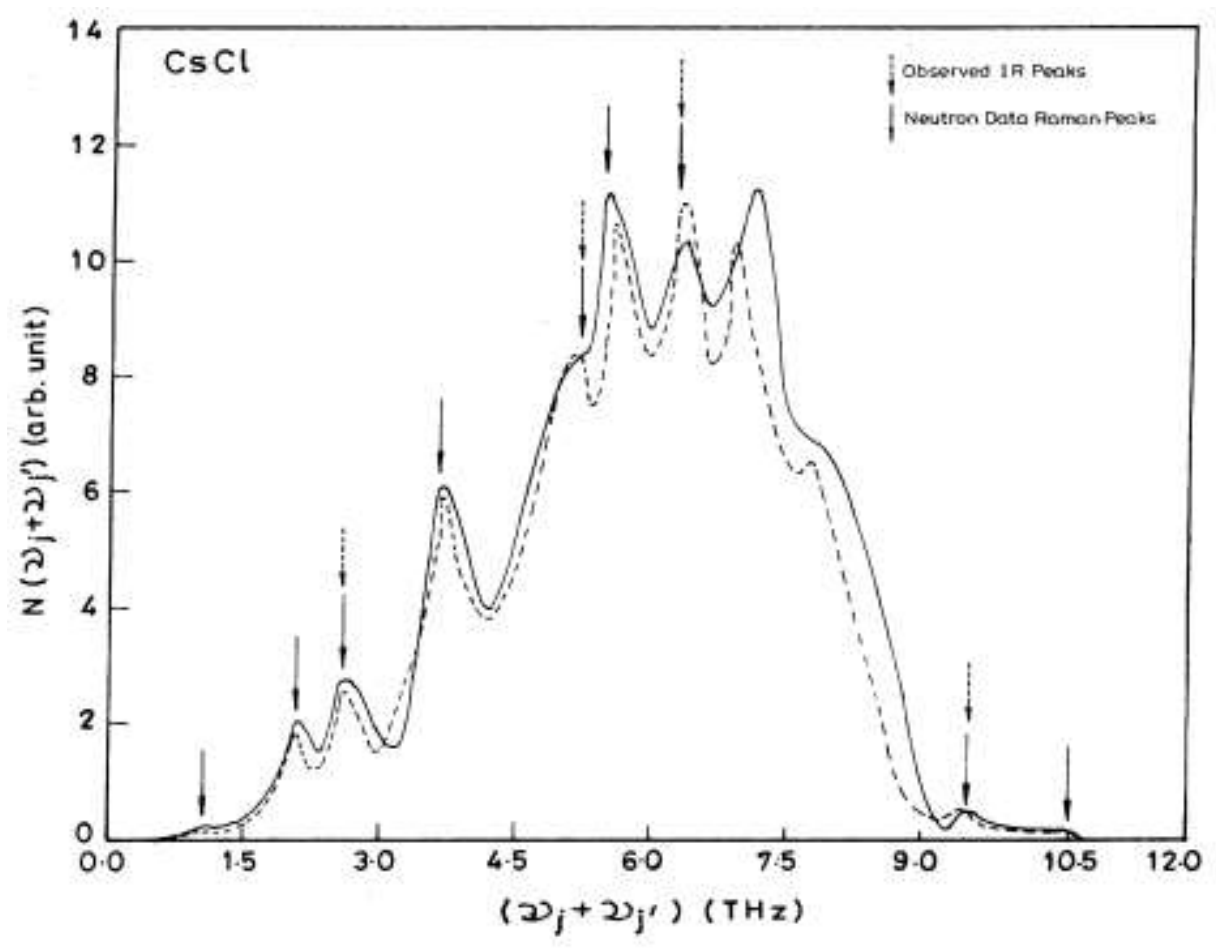

Figure 3: Combined density States (Two phonon) Curve for CsCl . $78 \mathrm{~K}$, $298 \mathrm{~K}$

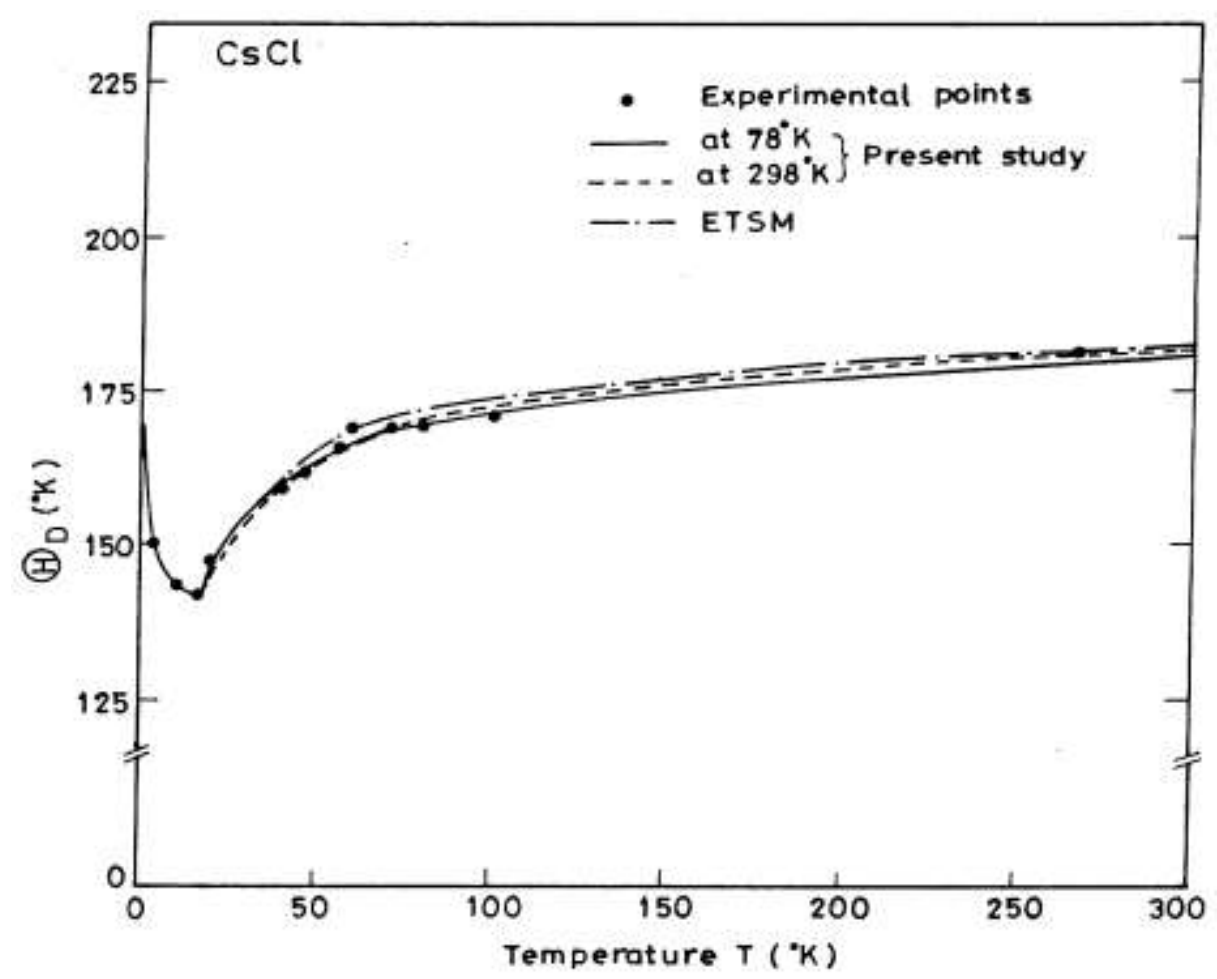

Figure 4: Debye Temperature Variation Curve for CsCl.

\section{Acknowledgements}

The authors are thankful to Prof. K.P.Mishra, VC and Dr. S.C. Tiwari, Pro. V.C., NGBU, Allahabad (India) for providing the necessary facilties. We are also thankful to Computer Center, BHU, Varanasi, India for providing computational assistance. 


\section{References}

[1]. Singh R.K., Gupta H.N. and Agrawal M.K., Phys. Rev. B 17, No. 2, 894 (1978).

[2]. Gupta H.N. and Upadhyaya R.S., Phys. Stat. Sol. (b) 102, 143 (1980).

[3]. Mishra V., Sanyal S.P. and Singh R.K., Philosophical Magazine A 55, No. 5, 583 (1987).

[4]. Reinitz K., Phys. Rev. 123, 615 (1961).

[5]. Lowndes R.P. and Martin D.H., Proc. R. Soc. Lond. A 308, 473 (1969).

[6]. Ahmad A.A.Z., Smith H.G., Wakabayashi N. and Wilkinson M.K., Phys. Rev. B 6, No. 10, 3956 (1972).

[7]. Rolandson S. and Raunio G., Phys. Rev. B4, No. 12, 4617 (1971).

[8]. Bührer W. and Hälg W., Phys. Stat. Sol. (b) 46, 679 (1971).

[9]. Taylor A.H., Gardner T.E. and Smith D.F., U.s. Bur. Mines Rept. Invest. No. 6157 (1963)

[10]. Sorai M., J. Phys. Soc. Japan 25, No. 2, 421 (1968).

[11]. Marshall B.J. and Kunel J.R., J. Appl. Phys. 40, 519 (1969).

[12]. Narayanan P.S., Proc. Ind. Acad. Sci. A42, 304 (1955).

[13]. Krishnamurthy N. and Krishnan R.S., Ind. J. Pure Appl. Phys. 1, 239 (1963).

[14]. Stekhnov A.I. and Korol'kov A.P., Sov. Phys. Solid State 8, 734 (1966).

[15]. Stekhnov A.I. and Korol'kov A.P., Sov. Phys. Solid State 4, 2311 (1963).

[16]. Puri D.S., Garg V.K. and Verma M.P., Phys. Stat. Sol. (b) 78, 113 (1976).

[17]. Garg V.K., Puri D.S. and Verma M.P., Phys. Stat. Sol. (b) 82, 325, 481 (1977).

[18]. Vetelino J.F., Mitra S.S., Phys. Rev., 178, 1349 (1969); Sol. Stat. Commun. 7, 1181 (1969).

[19]. Karo A.M. and Hardy J.R., J. Chem. Phys. 48, 3173 (1968).

[20]. Schroder U., Sol. Stat. Commun. 4, 347 (1966).

[21]. Basu, A.N. Sengupta S., Phys. Stat. Sol. 29, 367 (1968).

[22]. Lal H.H. and Verma M.P., J. Phys. C 5, 543 (1972).

[23]. Woods A.D.B., Cochran W. and Brockhouse B.N., Phys. Rev. 119, p 980 (1960).

[23]. Mishra V., Sanyal S.P. and Singh R.K., Pramana J. Phys. 28, No. 2, 217 (1987).

[25]. Kellermann E.W., Phil. Trans. Roy. Soc. (London) A 230, p 513 (1940).

[26]. Dick B.G. and Overhauser A.W., Phys. Rev. 112, p 90 (1958).

[27]. Verma M.P. and Singh R.K., Phys. Stat. Sol. 33, p 769 (1969); 36, p 335 (1969); 38, p 851 (1970).

[28]. Hafemeister D.W. and Flygare H.W. ,J. Chem. Phys. 43, 795 (1965).

[29]. Burstein E., Johnson F.A. and Loudon R., Phys. Rev. 139, p 1239 (1965).

[30]. Chang Z.P. and Barsch G.R., Phys. Stat. Sol. 23, 577 (1967).

[31]. K*ohler U, Johannsen P G and Holzapfel W B J. Phys.: Condens. Matter 9, 5581 (1997).

[32]. Upadhyaya K.S., Pandey Atul and Srivastava D.M., Chinese Journal of Phys., Vol. 44, 127 (2006).

[33]. Tiwari S.K., Pandey L.K., Shukla L.J., Upadhyaya K.S., Physica Scripta, 80, 1 (2009).

[34]. Tiwari S.K., Shukla L.J. and Upadhyaya K.S., Pramana Jour. Of Phys. 74, 793 (2010)

[35]. Tiwari S.K., Singh R.K., Upadhyaya K.S., Philosophical Magzine, 90, 3531(2010).

[36]. Srivastava U.C., Pandey R.S., \& Upadhyaya K.S., Int. Jour. Phys. Sci. 5(7), pp 972 (2010).

[37]. Srivastava U.C \& Upadhyaya K S, Rapid Communication (OAM - RC), Vol 4, ISS 9, pp 1336 (2010).

[38]. Srivastava U.C.\& Upadhyaya K. S., Phys. Rev. and Research Int. -1 (1),16-28. (2011).

[39]. Mishra K.K, Upadhyay G.K. and.Upadhyaya K.S, Phys. Rev. and Research Int. 2(2), 91-106, 2012.

[40]. Mishra K.K., Upadhyaya K.S., Int. Jour. of Sci. Engg. Res. 3 (8), 1-9, (2012)

[41]. Trivedi R.K..,Mishra K.K., Upadhyay S.K., Upadhyay G.K. and Upadhyaya K.S. IOSR Jour. Appl. phys.1(2), 24-32 (2012)

[42]. Mishra K.K., and Upadhyaya K.S., Int. Jour. of Engg. Res. Tech. 1 (5), 1 -3 (2012)

[43]. Mishra K.K and.Upadhyaya K.S, Physica Scripta (2012). 